\title{
Actitud hacia al aborto en estudiantes universitarios
}

\section{Attitude Toward Abortion in University Students}

\author{
Julio César Huamani Cahua ${ }^{1 *}$ \\ Universidad Católica San Pablo
}

Recibido: 07 - 10 - 17

\author{
Alison Serruto Castillo \\ Universidad Nacional de San Agustín
}

Aceptado: $21-11-17$

\section{Resumen}

En este estudio exploramos la actitud hacia el aborto en 400 estudiantes de cuatro universidades de la ciudad de Arequipa (100 estudiantes por universidad). El instrumento utilizado fue la Escala de Actitud hacia el Aborto de García (1990). Se obtuvo un valor promedio de 67.833 puntos, que indica una actitud favorable hacia el aborto. Asimismo, no se encontraron diferencias con respecto a la edad, género ni universidad de origen; sin embargo, existen diferencias significativas $(\mathrm{p}<0.05)$ según carrera profesional. Asimismo, las variables investigadas no explican la actitud hacia el aborto $\left(\mathrm{p}>0.1293 ; \mathrm{R}^{2}=0.0434\right)$.

Palabras clave: Actitudes; aborto; bioética; estudiantes universitarios.

\begin{abstract}
In this study we explored the attitude towards abortion in 400 students of four universities in the city of Arequipa (100 students per university). The instrument used was the Garcia Abortion Attitude Scale (1990). It was found that the average of 67,833 points indicates a favorable attitude toward abortion. Likewise, no differences were found regarding age, gender or university of origin; However, there were significant differences $(\mathrm{p}<0.05)$ according to career. Likewise, the variables investigated did not explain the attitude towards abortion ( $p>$ $\left.0.1293 ; \mathrm{R}^{2}=0.0434\right)$.
\end{abstract}

Key words: Attitude; abortion; bioethics; university students.

1 Universidad Católica San Pablo. Perú. *Correspondencia: jchuamani@ucsp.edu.pe 2 Universidad Nacional de San Agustín, Perú, alisonserruto@yahoo.es

(C) Los autores. Este artículo es publicado por la Revista de Investigación en Psicología de la Facultad de Psicología, Universidad Nacional Mayor de San Marcos. Este es un artículo de acceso abierto, distribuido bajo los términos de la licencia Creative Commons Atribucion - No Comercia_Compartir Igual 4.0 Internacional. (http://creativecommons.org/licenses/by-nc-sa/4.0/) que permite el uso no comercial, distribución y reproducción en cualquier medio, siempre que la obra original sea debidamente citada. 
La actitud hace referencia a la valoración positiva o negativa a través de la cual las personas juzgan cualquier aspecto de la realidad, que habitualmente se designa como objeto de actitud (Eagly \& Chaiken, 1998). Son además, elementos fundamentales en la construcción de representaciones sociales y se tornan indispensables para la estructuración del conocimiento de la realidad, teniendo estrecha relación con los valores culturales (Parales y Vizcaino, 2007).

Las actitudes se manifiestan en todas las personas y respecto a distintos temas. Ocurre lo mismo en torno al aborto, entendido como la interrupción del embarazo; sin embargo, no podría definirse como "interrupción", pues ello implicaría el poder reanudar posteriormente el proceso de gestación, situación que no es posible en caso de aborto (Márquez y Laje, 2016).

Por ello, preferimos definir el aborto, como la privación del nacimiento o una situación donde el parto no se ha concretado (Sánchez, 2011). No obstante, es posible distinguir entre el aborto espontáneo y el inducido. En el primer caso, el aborto ocurre por causas naturales, como consecuencia de una diversidad de factores que comprenden la vulnerabilidad física y psicológica de la madre. Entre los principales factores de riesgo se pueden mencionar, una implantación inadecuada del trofoblasto, el consumo de sustancias psicoactivas, la obesidad, deficiencias nutricionales, infecciones de transmisión sexual, malformaciones del útero, fibromas, etc. (Schwartz, 2003). En el segundo caso, el aborto es provocado de manera deliberada, por diversos motivos de índole psicosocial, pero que en la mayoría de los casos tiene que ver con la percepción del embarazo como una experiencia negativa, porque se contrapone a los intereses, metas y proyectos de la madre, del padre, o de ambos; o bien porque la madre tiene que afrontar su gestación sin la compañía y/o el apoyo del padre.

Debido a la complejidad de estos casos, las personas se encuentran enfrentadas ante lo siguiente: necesitan conseguir un determinado fin, pero se dan cuenta de que la acción por la cual pretenden alcanzarlo también producirá o podría producir un efecto negativo o moralmente malo. En ese sentido, el principio de razonamiento práctico que sirve para determinar la legitimidad o ilegitimidad de un acto que produce o puede producir dos efectos, de los cuales uno es bueno y el otro es malo, es denominado el principio de doble efecto (Miranda, 2008). En ese sentido, el aborto implica poner término a una vida humana, cuestión que viola los principios éticos de conservar y proteger la vida, más aún si los móviles del aborto tienen que ver con aspectos propios de la conveniencia de dicha actividad. Basados en esto, el aborto no tendría justificación moral, a no ser que hayan condiciones de riesgo vital, insalvables, para la madre (Muñoz y Neira, 2014).

En términos legales, en el Perú el aborto es penalizado, excepto en caso de amenaza grave a la vida o salud de la mujer. Así pues, los artículos del 114 al 120 del Código Penal contienen especificaciones e incluyen las consecuencias 
que conlleva la práctica del mismo. Sin embargo, a pesar de estas disposiciones legales, se ha evidenciado que la prevalencia de aborto es similar a la de aquellos países donde es legal, y hasta puede decirse que existe incremento de la frecuencia estimada de abortos (Palomino, Padilla, Talledo, Mazuelos, Carda y Bayer, 2011). Incluso se ha reportado la oferta en Internet de servicios clandestinos de aborto en el país (Merino-García, Meléndez y Taype-Rondán, 2015).

El estado del arte refleja que los estudios psicológicos en torno al aborto inducido han demostrado la relación causal entre variables sociodemográficas como sexo (Lara, 1987; García, Lara y Goldman, 2003), edad (Cabezas, Langer, Álvarez y Bustamante, 1998; Fernández, 2010), escolaridad (Serrano, 2011), estado civil (Távara y Sacsa, 2008) y norma grupal (Calderón y Alzamora, 2009) como determinantes de la actitud hacia el aborto inducido.

En ese sentido, la actitud hacía el aborto está relacionada con la situación tanto personal como familiar, correspondiente a la actualidad, pero también a su proyección futura, en la cual la participación de la mujer se percibe como fundamental (Cruz, 2012). Al respecto, cuando la actitud es favorable (Rojas, González, Padilla, Lavaut y Fuentes, 2012), lo es aún más en circunstancias particulares como violación, malformaciones, riesgo para la madre, etc. (López y Carril, 2010; Calatrava, Ruiz, López del Burgo, Irala y Osorio, 2009). Esta posición favorable se acentúa más en aquellas mujeres que son hijas de madre o padre adolescente (Doblado, De la Rosa y Junco, 2010). Sin embargo, de no darse en estas circunstancias la actitud hacia el aborto suele ser negativa (López y Carril, 2010).

Por otra parte, existe evidencia de actitud desfavorable hacia el aborto tanto en varones como mujeres (Chávez, Petrzelova y Zapata, 2009; Grajales y Cardona, 2012; Caneva, 2012). Particularmente en mujeres, predomina la actitud reactiva frente al aborto, la cual se caracteriza por una rotunda posición negativa hacia la práctica abortiva, así también se manifiesta actitud intolerante, la cual varía ante la presencia de circunstancias consideradas como atenuantes.

Los estudios en su mayoría están orientados a la figura femenina, sin embargo, también se registran datos acerca de lo que ocurre en el caso de los varones, para quienes el aborto sería algo más simbólico (Walzer, 1994), en tanto se entiende que es probablemente percibido como algo que le sucede a otra persona (Mora, 2001). Al respecto, Gutiérrez, Safora y Rodríguez (2009), encontraron principalmente un alto porcentaje de desinformación respecto al riesgo que conlleva un aborto para la pareja, asimismo, se evidenció la creencia de que los riesgos aparecen solo después de practicar el primer aborto. Además de ello, se considera como causa principal para el aborto, el abandono de la pareja y en segundo grado de importancia la interferencia con el proyecto de vida de uno o ambos padres. En ambas circunstancias la actitud hacia el aborto se torna favorable. En cambio, se 
evidencia intolerancia ante el aborto cuando el embarazo esta dado sin ninguna complicación física o psicológica; así también, se acompaña de una actitud reactiva, es decir, de rotunda negación al aborto sin mayor explicación (Caneva, 2012).

Existe evidencia de que los varones en su mayoría se oponen al aborto, pero se registra mayor aceptación para aquel que se daría en situaciones en las que la mujer tiene poco o ningún control sobre su condición, como cuando el embarazo es resultado de una violación, o cuando está en peligro la vida de la mujer (Bogess, \& Bradner, 2000; Adebayo, 1990; Misra, 1998). No obstante, en algunos países la admisión del aborto es más generalizada, como lo es en el caso de Dinamarca (Norup, 1997) y Suecia (Holmberg \& Wahlberg, 2000). Por otra parte, son los católicos y personas pertenecientes a otras religiones quienes aprueban en menor medida el aborto (Petracci, 2007; Presvelou, Brutus-García y Canivet-Gilson, 1972; Scott, 1989; De Boer, 1978; Adebayo, 1990; Marsiglio \& Shenan, 1993; Stone \& Waszak, 1992; Welzer, 1994; Hertel \& Russell, 1999). Sin embargo, no se encuentra relación entre religiosidad y actitudes hacia el aborto en los hombres del sur de Brasil (Fachel Leal, 1998).

Al respecto, ante la posibilidad de un embarazo, las motivaciones aducidas por los varones para favorecer un aborto son diversas, dentro de ellas: edad (demasiado joven o demasiado viejo para ser padre), ingresos insuficientes o situación de vivienda precaria (Dragestein, 1994). En el contexto nacional, se aduce con frecuencia que un hijo no planificado o fuera del matrimonio, es una carga en la vida o un deshonor para la mujer (Cáceres, 1998). En ese sentido, en una investigación realizada en Suecia, Kero, Lalos, Hogberg \& Jacobsson (1999), encontraron que la principal motivación que tienen los varones para aceptar un aborto era el deseo de tener hijos solamente dentro de un contexto familiar.

En la realidad peruana se ha señalado que predomina la actitud positiva hacia el aborto (Freitas y Pisco, 2013; Trujillo y Sembrera, 2016), no obstante, (Vélez, 2014) identificó una actitud desfavorable, tomando en cuenta los afectos implicados en la decisión de abortar, así como las acciones que se tomarían al respecto. Asimismo, existe gran diferencia respecto al sexo, ya que la funcionalidad familiar de las adolescentes mujeres influirá en torno a su actitud ante a un aborto; no ocurriendo así en caso del adolescente varón, pues la funcionalidad de la familia a la que pertenece, no tiene incidencia significativa.

Existe desacuerdo con la legalización del aborto, sin embargo, la actitud es favorable cuando se presentan situaciones como: malformaciones, peligro grave de la vida materna, violaciones, problemas económicos y embarazo no deseado; no obstante, a medida que se avanza en grado de estudios, la actitud se torna desfavorable casi sin excepción. De manera general, la mayoría de estudiantes no optaría por el aborto en caso de embarazo (Álvarez, 2015); así pues, mientras más alto es el nivel educativo, incrementa también la actitud de rechazo hacia el aborto 
inducido y en tanto sea más bajo es el nivel educativo la actitud de aceptación es mayor (Trujillo y Sembrera, 2016). Por lo expresado, se plantea la interrogante ¿Existe actitud desfavorable hacia el aborto en estudiantes universitarios?, En ese sentido, el objetivo de la investigación es identificar la actitud hacia el aborto, de los estudiantes universitarios de la ciudad de Arequipa según edad y sexo.

\section{MÉTODO}

\section{Participantes}

El estudio se realizó con una muestra de 400 estudiantes, provenientes de cuatro universidades de la ciudad de Arequipa; cada una con 100 estudiantes de sus distintas escuelas profesionales, seleccionadas accidentalmente. El muestreo casual o accidental es un método de muestreo no probabilístico donde los individuos se eligen de manera casual, sin ningún juicio previo (Rodríguez, 1993). En ese sentido, la recolección de los datos se realizó a los estudiantes de las distintas universidades, sin buscar comparar el tipo de carrera profesional, ni la universidad de origen, sino, realizar una elección representativa de las unidades de análisis de las distintas universidades de la ciudad de Arequipa.

La muestra de estudio estuvo conformada por 167 estudiantes varones y 233 estudiantes mujeres. La edad de los varones estuvo comprendida entre 17 y 31 años $(M=21.60 ; \mathrm{DE}=3.03)$, la edad de las mujeres fue de 16 a 28 años $(\mathrm{M}=20.52$; $\mathrm{DE}=2.31)$. Hubo diferencias con respecto al sexo de los participantes $\left(\mathrm{t}_{398}=3.889\right.$; $\mathrm{p}=0.000)$ para varianzas diferentes.

\section{Instrumentos}

El instrumento utilizado es la Escala de Actitud hacia el Aborto de García (1990), que consta de 31reactivos en una escala tipo Likert que evalúan la actitud hacia el aborto, con 4 opciones de respuesta que van desde "Totalmente en desacuerdo" hasta "Totalmente de acuerdo". Cada ítem tiene un puntaje de 1 a4 según la respuesta que indique el evaluado, respectivamente, de tal modo que, cuanto más alto es el puntaje mayor es el acuerdo con la premisa. El instrumento fue sometido al análisis factorial exploratorio con base al método de componentes principales y al método de rotación varimax, presentando un valor de adecuación al muestreo de Kaiser-Mayer-Olkin de 0.921 y un test de esfericidad de Bartlett que fue significativo $(\mathrm{p}<0.00)$, lo cual garantiza la pertinencia de la ejecución del análisis factorial exploratorio. Los resultados indican la existencia de un solo factor, el cual permite explicar el $38,78 \%$ de la varianza total y que implicaría que la prueba es unidimensional.

El análisis de la confiabilidad de la Escala de actitud hacia el aborto se realizó mediante el método de consistencia interna a través del Coeficiente Alfa de Cronbach, obteniéndose un coeficiente de confiabilidad 0.671 considerado como 
regular y que nos dice que la Escala de actitud hacia el aborto ofrece medidas confiables.

\section{Procedimientos}

Los estudiantes fueron evaluados en sus respectivas universidades, durante el horario de funcionamiento de dichas instituciones. Los estudiantes dieron su consentimiento para el llenado del instrumento, dándoles información de los fines del estudio.

\section{RESULTADOS}

Los resultados indican que la actitud hacia el aborto es favorable en los estudiantes investigados, encontrando que el 50\% de los estudiantes tienen puntajes entre 64 y 71 puntos, tal como se aprecia en la tabla 1 .

\section{Tabla 1}

Análisis descriptivo numérico de la actitud hacia el aborto en estudiantes universitarios

\begin{tabular}{lccccccc}
\hline Variable & Obs. & Media & Err.std. & \multicolumn{2}{c}{ [95\% I.C.] } & Min & Max \\
\hline Actitud hacia el aborto & 400 & 67.83 & 30.272 & 67.298 & 68.367 & 37 & 89 \\
\hline
\end{tabular}

Con respecto a la actitud hacia el aborto según sexo, utilizando la prueba t de student encontramos que las varianzas son iguales (0.6574), asimismo, se observa que no existen diferencias estadísticamente significativas.

Tabla 2

Diferencias de medias a través de la t de student de la actitud hacia el aborto según sexo

\begin{tabular}{|c|c|c|c|c|c|}
\hline Grupo & Obs. & Media & Err.std. & Desv. Std. & 95\% I.C. \\
\hline Hombre & 167 & 67.689 & 0.431 & 5.575 & $66.837 \quad 68.541$ \\
\hline Mujer & 233 & 67.936 & 0.350 & 5.347 & $67.245 \quad 68.626$ \\
\hline Combinado & 400 & 67.832 & 0.272 & 5.438 & $67.298 \quad 68.367$ \\
\hline
\end{tabular}

$(\mathrm{F}>\mathrm{f})=0.5560$

El ANOVA de un factor, indica que no existen diferencias en la actitud hacia el aborto según tipo de universidad, considerando que se analizaron 100 encuestas aplicadas a estudiantes de cada una de las cuatro universidades más representativas de la ciudad de Arequipa.Las medias para las universidades en los subconjuntos homogéneos son: $\mathrm{UCSP}=69,58$; $\mathrm{UCSM}=70,18 ; \mathrm{UAP}=71,94$ y $\mathrm{UNSA}=72,48$. 
Tabla 3

ANOVA de un factor para la actitud hacia el aborto según universidad de origen

\begin{tabular}{lccccc}
\hline Fuente & SC & df & MS & F & Prob $>$ F \\
\hline Entre grupos & 115.508 & 3 & 38.503 & 1.3 & 0.272 \\
Intra grupos & 11684.27 & 396 & 29.506 & & \\
Total & 11799.775 & 399 & 29.573 & & \\
\hline
\end{tabular}

Para realizar el análisis de regresión lineal múltiple cumpliendo los supuestos de normalidad ( $\mathrm{p}>0.05$ ), se utilizó el método de Mínimos Cuadrados Ordinarios (MCO), por ser un estudio transversal. El modelo propuesto fue:

$$
\text { Actitud hacia el aborto }=b o+b 1(\text { edad })+b 2(\text { sexo })+e
$$

Se observa que $\left(\operatorname{Prob}>\mathrm{F}=0.1293 ; \mathrm{R}^{2}=0.043\right)$ este coeficiente indica que no existe relación significativa, es decir, la edad ni el sexo explican la actitud hacia el aborto.

Tabla 4

Análisis de regresión lineal múltiple de la edad y el sexo que explican la actitud hacia el aborto

\begin{tabular}{|c|c|c|c|c|c|c|c|c|}
\hline \multirow{2}{*}{$\begin{array}{l}\text { Actitud hacia el } \\
\text { aborto }\end{array}$} & \multirow{2}{*}{ Coef. } & \multirow{2}{*}{$\begin{array}{l}\text { Std. } \\
\text { Error }\end{array}$} & \multirow{2}{*}{ t } & \multirow{2}{*}{$\mathbf{P}>\mathbf{T}$} & & & \multirow{2}{*}{ P valor } & \multirow{2}{*}{ R-squared } \\
\hline & & & & & \multicolumn{2}{|c|}{ Conf. Interval. } & & \\
\hline Edad & 0.360 & 0.203 & 1.77 & 0.077 & -0.039 & 0.758 & & \\
\hline Sexo & -1.249 & 1.081 & -1.16 & 0.249 & -3.374 & 0.876 & 0.1293 & 0.043 \\
\hline Constante & 59.089 & 4.383 & 13.48 & 0.000 & 50.471 & 67.706 & & \\
\hline
\end{tabular}

\section{Discusión}

Los resultados encontrados indican una actitud favorable hacia el aborto en los estudiantes universitarios, es decir, la valoración que realizan respecto a la práctica abortiva es positiva. En tal sentido, Freitas y Pisco (2013), Trujillo y Sembrera (2016), evidenciaron que la actitud favorable se manifestaba solo en caso de malformaciones genéticas, violación, dificultades económicas, peligro grave de la vita materna, y embarazo no deseado (Álvarez, 2015). Ello se complementa con los trabajos de López y Carril (2010) y Calatrava et al. (2009), los cuales corresponden a la realidad de otros países.

En los estudiantes universitarios el tema del aborto específicamente no ha sido tratado de manera interdisciplinaria y en las investigaciones registradas no existen resultados que lleguen a consensos. No obstante, existen mayores datos respecto al embarazado no deseado, se sabe que el estrés ocasionado por 
una gestación no deseada se manifiesta en cambios neurobiológicos, como por ejemplo la reducción del factor neurotrófico derivado del cerebro (BDNF), que es un marcador biológico de la depresión (Rondón, 2015). Asimismo, se ha indicado que cuando una mujer tiene un embarazo no deseado, la probabilidad de que pueda desarrollar problemas de salud mental después de ese embarazo es similar tanto si permite el nacimiento como si recurre al aborto (Felipe, 2014). Se ha evidenciado, a través de la investigación en la trayectoria de los síntomas depresivos, que entre el embarazo planificado, el inoportuno pero aceptado y el no deseado; es el embarazo no deseado (aquel que la mujer no desea ni acepta, no solamente el que se presenta sin estar planificado) el que duplica el riesgo de depresión posparto para la mujer (Mercier, Garrett, Thorp \& Siega-Riz, 2013). En ese sentido, es probable que la interpretación que realizan los estudiantes de esta información se relacione con la postura que asumen ante el aborto, pues para que se tome una actitud con relación a un tema, es necesario que se tenga alguna representación cognoscitiva del mismo (Vélez, 2014). A la luz de estos datos; resulta relevante poner mayor énfasis en la comprensión de una problemática tan compleja como la del embarazo no deseado y la sexualidad humana, así como del aborto y sus implicancias éticas, psicológicas, familiares y sociales.

En ese sentido, es importante tomar en cuenta que aspectos como la diversidad de características femeninas, así como las circunstancias y razones que manifiestan para evitar un nacimiento, no se han incorporado adecuadamente dentro de la teoría o investigación que busca dar explicación a la variación en la salud mental posterior un aborto. Del mismo modo, los sesgos de muestreo selectivo, la presencia de factores comunes de riesgo y el hecho de confundir el aborto con un embarazo no deseado, son limitaciones que están aún presentes al momento de realizar investigación en torno a este tema.

Se ha argumentado significativamente respecto a si las reacciones emocionales de las mujeres que se han provocado un aborto tienen un patrón definido y generalizable para todas. La verdad es que frente a esto no hay consenso, sin embargo, las consecuencias psicológicas encontradas se han descrito bajo el nombre de Síndrome Post Aborto (Speckhard \& Rue, 1992), las mismas que han sido corroboradas en relación al aborto terapéutico (Rosenfeld, 1992) y por autores del área de fertilización asistida (Garek, 1995). Este síndrome no es reconocido por el manual DSM-V, ni por todos los profesionales de la práctica clínica, pero es muy similar y manifiesta casi todas las características del trastorno por estrés postraumático. No obstante, las inferencias realizadas al respecto se consideran insuficientes desde el punto de vista estadístico (Major, Applebaum, Beckman, Dutton, Russo \& West, 2008).

En ese sentido, cabe mencionar que al analizar las búsquedas más recurrentes en relación al tema del aborto, se evidenció que en la población peruana existe un creciente interés por saber cómo abortar y cuáles son las pastillas utilizadas 
(Tejada, 2016), lo cual resulta sumamente preocupante si se considera que existen reportes de inducción del aborto de manera clandestina en nuestro país (Palomino et al., 2011), que terminan no solo por aniquilar la vida del ser humano por nacer, sino que también ponen en riesgo significativo la vida de la gestante.

No obstante, Chávez, Petrzelova y Zapata (2009), Grajales y Cardona (2012) y Caneva (2012) señalan la predominancia de actitud desfavorable hacia el aborto. En otros casos, dicha actitud solo se presenta cuando el embarazo esta dado sin ninguna complicación física o psicológica (López y Carril, 2010). En la realidad peruana, Veléz (2014) corroboró esta posición.

Esta contradicción en los resultados de las diversas investigaciones, se debe probablemente a que las poblaciones de estudio provienen de contextos distintos en términos culturales y legales. Al analizar la actitud hacia en aborto según sexo y edad se evidencia que no existen diferencias significativas, ello se corrobora con los estudios tanto nacionales como internacionales (Freitas y Pisco, 2013; Trujillo y Sembrera, 2016; Álvarez, 2015; López y Carril, 2010; Calatrava et al., 2009; Petrzelova y Zapata, 2009; Grajales y Cardona, 2012; Caneva, 2012 y Velez, 2014). Al respecto, Doblado, De la Rosa y Junco (2010) señalan que las mujeres hijas de madres o padres adolescentes tienen una actitud más favorable, y Caneva (2012) señala que los varones tienen una actitud intolerante hacia el aborto, cuando el embarazo no presenta ningún tipo de complicación o riesgo.

En ese sentido, cabe mencionar que se ha encontrado relación entre el nivel de conocimientos y la actitud hacia el aborto: así pues, mientras más alto sea el nivel educativo habrá mayor actitud de rechazo hacia el aborto inducido y en caso contrario, es decir, cuando el nivel educativo es bajo se manifiesta un incremento en la actitud de aceptación (Trujillo y Sembrera, 2016; Álvarez, 2015). De esto se desprende la necesidad de brindar una educación pertinente y accesible a diversos sectores de la población, que aborde el tema del aborto, en salvaguarda de la vida, poniendo como base los valores morales.

Finalmente, los resultados obtenidos deberán servir como base para futuras investigaciones que consideren otras variables y permitan ampliar el conocimiento respecto a este tema en la realidad peruana. Más aún si los resultados, nos presentan una preocupante situación, con respecto a sus actitudes frente a un tema que suele ser manipulado por ciertos grupos de interés que se manifiestan abiertamente en contra de la vida.

\section{REFERENCIAS}

Adebayo, A. (1990). Male attitudes toward abortion: an analysis of urban survey data. Social Indicators Research, 22(2), 213-228.

Álvarez, J. (2015). Actitudes y conocimientos frente al aborto en estudiantes de la EAP de Obstetricia de la Facultad de Medicina de San Fernando.UNMSM-2014. (Tesis 
de grado). Recuperado el 1 de marzo de 2017 de: http://www.alicia.concytec.gob.pe/ vufind/Record/UNMSM_e0322941913f43d5ae14c35b79084119b

Bogess, S. \& Bradner, C. (2000). Trends in adolescent male's abortion attitudes, 19881995: differences by race and ethnicity. Family Planning Perspectives, 32(3), 118-123.

Cabezas, E., Langer, A., Álvarez, L. y Bustamante, P. (1998). Perfil sociodemográfico del aborto inducido. Salud Pública de México, 40, 265-272.

Cáceres, C. (1998). Jóvenes varones en Lima: dilemas y estrategias en salud sexual. En Valdés, T., y Olavarría, J. (Eds.), Masculinidades y equidad de género en América Latina (pp. 158-174). Santiago de Chile: FLACSO - UNFPA.

Calatrava, M., Carlos, S., Ruiz, M., López del Burgo, C., Irala, J. y Osorio, A. (2009). Opiniones de estudiantes universitarios sobre el aborto. Cuadernos de Bioética, 20(3), 527-528.

Calderón, J. y Alzamora, L. (2009). Factores de pareja y grupales relacionados al aborto provocado en mujeres adolescentes. Scientia, 1, 110-116.

Caneva, H. (2012). Representaciones sobre el aborto. Estudio de jóvenes de sectores pobres de la ciudad de La Plata.VII Jornadas de Sociología de la Universidad Nacional de La Plata "Argentina en el escenario latinoamericano actual: debates desde las ciencias sociales". Recuperado el 1 de marzo de 2017 de: http://jornadassociologia. fahce.unlp.edu.ar

Chávez, M., Petrzelová, J., y Zapata, J. (2009). Actitudes respecto a la sexualidad en jóvenes universitarios. Entrevista e Investigación en Psicología, 14(1), 137-131.

Cruz, L. (2012). Estudio de actitudes hacia el aborto en estudiantes universitarios de Morelo México. Xihmai, 7(13), 61-83.

Cruz, L., Carreón, J., Hernández, J., Bautista, M. y Méndez, A. (2012). Determinantes socioeconómicos y demográficos de las actitudes hacia el aborto. Psicogente, 15(28), 262-270.

De Boer, C. (1978). The polls: abortion. Public Opinion Quarterly, 41(4), 553-564.

Doblado, N., De la Rosa, I. y Junco, A. (2010). Aborto en la adolescencia un problema de salud. Revista Cubana de Obstetricia y Ginecología, 36(3), 409-421.

Dragestein, B. (1994). Schwangerschftsabbruch: was empfi ndet der begleitende Partner. Pro Familia Magazine, 3, 12-17.

Eagly, A. H. \& Chaiken, S. (1998). Attitude structure and function. En D. T. Gilbert, S. T. Fiske, \& G. Lindzey (Eds.), The Handbook of Social Psychology (4th ed. Vol. 1, pp. 269-322). New York: McGraw-Hill.

Fachel Leal, O. (1998). Hombres y mujeres: cultura reproductiva y sexualidad en el sur de Brasil. Ponencia en la Conferencia regional La Equidad de género en América Latina y el Caribe: Desafios desde las identidades masculinas, 8-10 de julio, Santiago de Chile.

Felipe, N. (2014). Abortion, unwanted childbearing and mental health. Salud Mental, $37(4), 283-291$. 
Fernández, M., Carrillo, F., Leal, M., Carrillo, I., Carrillo, E., Lozano, M., Fernández, S. y Pastor, L. (2010). Una aproximación al aborto e inmigración. El valor socioeconómico de la vida humana. Cuadernos de Bioética, 3, 313-327.

Freitas, L. y Pisco, G. (2013). Conocimiento y actitudes hacia el aborto en usuarios del módulo de atención integral del adolescente hospital regional de Loreto Punchana2013. (Tesis de pregrado). Recuperado el 15 de marzo de 2017 de: http://www.alicia. concytec.gob.pe/vufind/Record/UPOU_a693b7bfaa5e9222ce1236df2c4065a7.

García, S., Lara, D. y Goldman, L. (2003). Conocimientos, actitudes y prácticas de los médicos mexicanos sobre el aborto: resultados de una encuesta nacional. Gaceta Médica Mexicana, 139, 91-104.

Garek, M. (1995). Psychological effects of embryonal reduction. From the decision making to 4 months after delivery. Journal of Gynecology Obstetrics and Human Reproduction, 24(2), 119-145.

González, A., Soriano, M. y Soriano, E. (2014). Educación para la salud sexual. Del enamoramiento al aborto. Un estudio cualitativo con adolescentes españoles e inmigrantes. Perfiles Educativos, 36(144), 105-119.

Grajales, I., y Cardona, D. (2012). Actitudes de los adolescentes escolarizados frente a la salud sexual y reproductiva. Medellín (Colombia). Revista de Investigación en Educación y Enfermería, 30(1), 77-85.

Gutiérrez, T., Safora, O. y Rodríguez, A. (2009). Percepción en el varón del aborto en la adolescencia. Revista Cubana de Obstetricia y Ginecología, 35(4), $171 \square 177$.

Hertel, B., \& Russell, M. (1999). Examining the absence of a gender effect on abortion attitudes: is there really no difference? Sociological Inquiry, 69(3), 364-381.

Holmberg, L., \& Wahlberg, V. (2000). The process of decision-making on abortion: A grounded theory study of young men in Sweden. Journal of Adolescent Health, 26, 230-234.

Kero, A., Lalos, A., Hogberg, U. \& Jacobsson, L. (1999). The male partner involved in legal abortion. Human Reproduction, 14(10), 2669-2675.

Lara, H. (1987). Premisas socioculturales, planificación familiar y aborto. Revista Sonorense de Psicología, 1, 26-36.

López, A. y Carril, E. (2010). Aborto voluntario y subjetividad en contextos de penalización. Efectos y significados en mujeres, varones y profesionales de la salud. Psicología, Conocimiento y Sociedad, 1(2), 1-32.

Major, B., Applebaum, M., Beckman, L., Dutton, M. A., Russo, N. F., \& West, C. (2008). Report of the APA Task Force on Mental Health and Abortion. Recuperado el 16 de enero de 2017 de: https://www.reproductiverights.org/sites/crr.civicactions.net/files/ documents/American\%20Psychological\%20Association--Mental\%20Health.pdf

Márquez, N. y Laje, A. (2016). El libro negro de la nueva izquierda. Buenos Aires: Grupo Unión. 
Marsiglio, W. \& Shenan, C.L. (1993). Adolescent males abortion attitudes: data from a national survey. Family Planning Perspectives, 25(4), 162-169.

Mercier, R., Garrett, J., Thorp, J. \& Siega-Riz, A. (2013). Pregnancy intention and postpartum depression: secondary data analysis from a prospective cohort. International Journal of Obstetrics and Gynaecology, 120(9), 1116-22.

Merino-García, N., Meléndez, W. \& Taype-Rondán, A. (2015). Abortion services offered via the Internet in Lima, Peru: methods and prices. Journal of Family Planning and Reproductive HealthCare, 42, 77-85.

Miranda, J. (2008). El principio del doble efecto y su relevancia en el razonamiento jurídico. Revista Chilena de Derecho, 35(3), 485 - 519.

Misra, R. (1998). Effect of age, gender and race on abortion attitudes. International Journal of Sociology and Social Policy, 18(910), 94-118.

Mora, M. (2001). Hombres colombianos. Ponencia presentada en el Encuentro Regional de Investigadores: El embarazo no deseado y el aborto inducido: desafíos de salud pública en América Latina y el Caribe. Organizado por el Population Council, Cuernavaca, México.

Muñoz, A. y Neira, J. (2014). Familias que conciben un hijo con malformación congénita letal. En. Red de Institutos Latinoamericanos de Familia, Congreso de Investigación Cientifica en Familia (pp. 37-44). Chile: REDIFAM.

Norup, M. (1997). Attitudes toward abortion in the Danish population. Bioethics, 11(5), 439-449.

Palomino, N., Padilla, M., Talledo, B., Mazuelos, C., Carda, J. y Bayer, A. (2011). The social constructions of unwanted pregnancy and abortion in Lima, Peru. Glob Public Health, 6(1), 73-89. doi: 10.1080 / 17441692.2011.590813

Parales, C. y Vizcaino, M. (2007).Las relaciones entre actitudes y representaciones sociales: elementos para una integración conceptual. Revista Latinoamericana de Psicología, 39(2), 351-361.

Petracci, M. (2007). Experiencias, representaciones y opiniones sobre aborto. Un estudio exploratorio en varones adultos de Buenos Aires. VI International Conference: Dis/ Organized Pleasures-Changing Bodies, Rights and Cultures, Lima, Perú, junio 2729.

Presvelou, C., Brutus-García, A., \& Canivet-Gilson, B. (1972). Lávortement dans la vie du couple. Recherches Sociologiques, 3(1), 144-160.

Rodríguez, J. (1993). Métodos de muestreo. Casos prácticos. Madrid: CIS

Rojas, I., González, R., Padilla, M., Lavaut, K. y Fuentes, L. (2012). Actitudes de individuos de la población cubana hacia el aborto selectivo. Memorias Convención Internacional de Salud Pública (pp. 3-7). La Habana: Progreso.

Rondón, M. (2015). Salud mental y aborto terapéutico. Anales de la Facultad de Medicina, $76(4), 407-411$. 
Rosenfeld, J. (1992). Emotional responses to therapeutic abortion. American Family Phyysician, 45(1), 137-177.

Sánchez, J. (2011). Análisis del Aborto Derivado de Casos de Violación Sexual dentro del Modelo Jurídico Vigente en el Perú: Una Aproximación desde los Fundamentos Filosóficos del Artículo Primero de La Constitución Política del Perú. (Tesis de grado). Pontificia Universidad Católica del Perú, Lima, Perú. Recuperado el 30 de marzo de 2017 de: file://C:/Users/HP/Downloads/SANCHEZ_PEREZ_JORGE_ HUMBERTO_ANALISIS_ABORTO.pdf

Schwartz, R. (2003). Obstetricia. 6ta. edición. Buenos Aires: Editorial Ateneo.

Scott, J. (1989). Conflicting beliefs about abortion: legal approval and moral doubts. Social Psychology Quarterly, 52(4), 319-326.

Serrano, M. (2011). Conocimiento, actitud y percepción de los médicos residentes de Ginecología y Obstetricia del ISSSTE acerca de la anticoncepción de emergencia. Revista de Especialidades Médico Quirúrgicas, 16, 215-220.

Speckhard, A. \& Rue, V. (1992). Postabortion syndrome: an emerging public health concern. Journal of Social Issues, 48(3), 95-120.

Stone, R. \& Waszak, C. (1992). Adolescent knowledge and attitudes about abortion. Family Planning Perspectives, 24(2), 52-57.

Távara,L.y Sacsa,D.(2008). Conocimientos, actitudes y prácticas demédicos ginecobstetras en relación al aborto. Centro de promoción de los derechos sexuales y reproductivos. Lima. Recuperado el 02 de enero de 2017 de: http:/www.clacaidigital. info: 8080/ xmlui/bitstream/handle/123456789/113/conocimientosyactitudesLuisTavara. pdf? sequence $=1$.

Tejada, P. (2016). Abortion internet search. An evaluation with google trend in Peru. Cartas al Director/Gaceta Sanitaria, 30(4), 318-321.

Trujillo, G. y Sembrera, E. (2016).Conocimientos y actitudes sobre el aborto inducido en adolescentes del 5 to año de secundaria de una institución educativa. Revista Apuntes Universitarios, 6(1), 47-60.

Vélez, K. (2014).Relación entre la funcionalidad familiar y la actitud según género frente al aborto en adolescentes de la institución educativa industrial $n^{\circ} 32$. (Tesis de grado).Recuperado el 13 de enero de 2017 de:http://www.alicia.concytec.gob.pe/ vufind/Record/UNPP_6911c5f088038c2c35b0b5985b833622

Walzer, S. (1994). The role of gender in determining abortion attitudes. Social Science Quarterly, 75, 687-693. 